\title{
BASF baut neues Forschungsgebäude
}

D

e BASF baut im Süden des Stamm-

werks Ludwigshafen in der Nähe von Tor 7 ein neues Laborgebäude, das auf 4300 Quadratmetern Fläche Materialphysikern sowie Additiv-, Formulierungs- und Polymerforschern beste $\mathrm{Vo}^{-}$ raussetzungen für eine noch engere Zusammenarbeit bieten soll. Die Investitionssumme beträgt rund 50 Millionen $€$.

Dazu Dr. Andreas Kreimeyer, Mitglied des Vorstands und Sprecher der
Forschung: „Innovationen auf Basis effizienter und effektiver Forschung und Entwicklung sind ein wichtiger Eckpfeiler unserer Strategie und Garant für organisches Wachstum. Mit dem neuen Gebäude sorgen wir dafür, dass der Standort Ludwigshafen international wettbewerbsfähig bleibt und stärken so unseren globalen Forschungsverbund“.

Die BASF legt beim Bau der neuen Laborräume, deren Bezug im zweiten

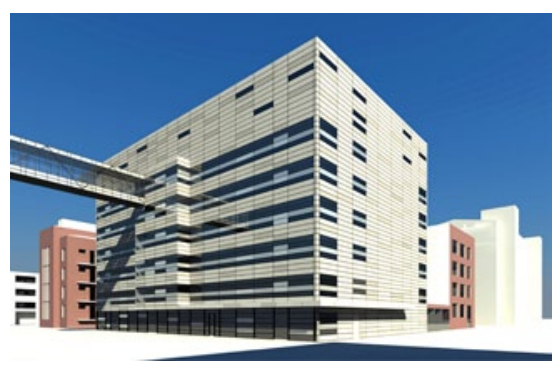

Halbjahr 2015 geplant ist, besonderen Wert auf den Einsatz von energieeffizienten Lösungen.

\section{Fraunhofer CBP: Industriechemikalien aus nachwachsenden Rohstoffen}

In Anwesenheit von Bundeskanzlerin Merkel wurde kürzlich nach knapp zwei Jahren Bauzeit in Jena das FraunhoferZentrum für Chemisch-Biotechnologische Prozesse CBP feierlich eröffnet. Dieses neue Institut soll die Lücke zwischen Labor und industrieller Umsetzung bei der Nutzung nachwachsender Rohstoffe schließen.

K lebstoffe, Kunststoffe oder Lacke sowie viele wichtige Zwischenprodukte der chemischen Industrie werden immer noch vorwiegend aus Erdöl hergestellt. Doch weltweit arbeiten Chemieunternehmen daran, Erdöl durch nachwachsende Rohstoffe zu ersetzen. Im Labormaßstab funktionieren einige dieser neuen Verfahren, die ohne Lebens- und Futtermittel auskommen sollen, schon sehr gut. Die Nutzung nachwachsender Rohstoffe in industriellen Dimensionen ist allerdings selbst für große Unternehmen ein erheblicher finanzieller und technologischer Kraftakt. Auch kleine und mittlere Unternehmen scheitern bisher oft daran, ihre Produkte in die technische Anwendung zu bringen, obwohl sie im Labor bereits erfolgreich attraktive Produkte entwickelt haben. Dies wird sich mit dem neuen Fraunhofer-Zentrum für Chemisch-Biotechnologische Prozesse CBP ändern, das am 2. Oktober 2012 nach nur knapp zwei Jahren Bauzeit feierlich eröffnet wurde.

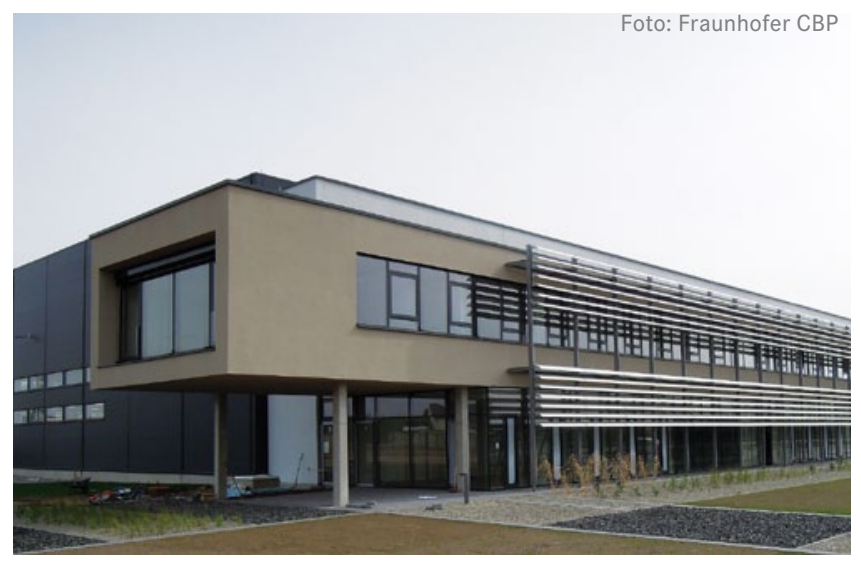

Neubau des Fraunhofer-Zentrums für Chemisch-Biotechnologische Prozesse CBP
„Das neue Fraunhofer-Zentrum für Chemisch-Biotechnologische Prozesse CBP schließt die Lücke zwischen Labor und industrieller Umsetzung bei der Nutzung nachwachsender Rohstoffe“, sagte Professor Reimund Neugebauer, der am 1. Oktober 2012 sein Amt als Präsident der Fraunhofer-Gesellschaft antrat. „Unsere produzierenden Unternehmen sind noch weitgehend von fossilen Rohstoffen abhängig. Mit regenerativen Rohstoffen können wir sowohl diese Abhängigkeit als auch die $\mathrm{CO}_{2}$-Emmissionen weiter reduzieren. Mit dem Fraunhofer CBP werden zudem Prozesse möglich, die vielfach rohstoff- und energieeffizienter als die petrochemischen Verfahren sind. Damit sind wir auf dem Weg zu einer nachhaltigen Chemie einen entscheidenden Schritt weitergekommen.“

Betrieben wird das Fraunhofer CBP gemeinsam von Wissenschaftlern der Fraunhofer-Institute für Grenzflächen- und Bioverfahrenstechnik IGB (Stuttgart) und für Chemische Technologie ICT (Pfinztal). Nach der zweijährigen Aufbauphase sind bereits 19 Mitarbeiterinnen und Mitarbeiter am CBP tätig.

Für die Entwicklung und Skalierung von biotechnologischen, chemischen sowie kombinierten Verfahren stehen fünf verschiedene Prozessanlagen nach dem Modell einer Bioraffinerie als separat zu betreibende oder je nach Bedarf einfach zu kombinierende Module bereit. Mit diesem sehr flexiblen Konzept bieten sich neue Möglichkeiten, um die Nutzung von biologischen Rohstoffen weiterzuentwickeln und bis in die industrielle Größenordnung voranzutreiben. Auf diese Weise wird es möglich, die etablierten Prozesse der chemischen Industrie mit neuen, innovativen Verfahren zu kombinieren und so beispielsweise öl-, lignin-, cellulose-, stärkeoder zuckerhaltige Rohstoffe als Ausgangsstoffe für Produkte zu gewinnen. 